\title{
MUTATION
}

\section{It's the $\mathrm{CpG}$ content that counts}

The rate of neutral mutations those that are not subject to natural selection - shows substantial variation, but the factors contributing to mutational rate have remained elusive. Now, new evidence indicates that the CpG content of DNA can influence the mutation rate of non-CpG-containing sequences, suggesting that the intrinsic properties of DNA sequences may be more important than the chromosomal environment in determining mutation rates and genome integrity.

In mammals, the Cs of most CpG base pairs are methylated and hypermutable, as methyl-C is spontaneously deaminated, producing a $\mathrm{T}: \mathrm{G}$ mismatch. Consequently, methylCpGs have a mutation rate that is 10-50 times greater than that of Cs in the context of other bases, and CpGs that are not subject to selection are replaced by TpG/CpA and eliminated from the genome. Walser and Furano took advantage of these properties of CpGs and compared the CpG contents of LINE-1 non-longterminal-repeat retrotransposons in human and chimpanzee genomes. As these retrotransposon sequences are not under selection, 'old' sequences should have a lower CpG content than 'younger' ones.

Intriguingly, previous work has shown that the correlation between
CpG content and neutral mutation rate holds for non-CpG-containing sites as well as for $\mathrm{CpG}$ sequences. How could this effect be explained? Two main explanations have been proposed: the chromosomal environment could influence the non-CpG mutation rate and $\mathrm{CpG}$ content or, alternatively, CpGs could directly affect flanking non-CpG sequences. Walser and Furano found that the correlation between non-CpG mutation rates and $\mathrm{CpG}$ content is best fitted by a sigmoidal function, suggesting that a threshold $\mathrm{CpG}$ content is needed to affect the non-CpG mutation rate and that a 'saturation point' can also be reached, after which further increases in $\mathrm{CpG}$ content have no effect on mutation rate. In addition, they found that the type of mutation is affected by the non-CpG mutation rate - there were changes in the ratio of transition mutations (purine to purine or pyramidine to pyramidine interchanges) to transversion mutations (purine to pyramidine interchanges) that correlated with the changes in nonCpG mutation rate. As different underlying mechanisms produce transitions and transversions, the authors suggest that their findings are more consistent with the hypothesis that $\mathrm{CpG}$ s have a direct mutational effect on surrounding non-CpG DNA.

How might CpGs influence mutation rates of non-CpG DNA? Given the propensity for methyl-CpGs to deaminate and produce mismatches, it is plausible that error-prone mismatch repair mechanisms may have a role. $\mathrm{CpG}$ methylation might also have epigenetic effects by promoting chromatin states that make DNA more susceptible to mutation. The findings of this study could also have potential implications for diseases, as the authors observed an increase in transversions in LINE-1 sequences, and increases in transversions have been associated with 'mutator phenotypes' in some cancers.

Meera Swami

ORIGINAL RESEARCH PAPER Walser, J. C. \& Furano, A. V. The mutational spectrum of non-CpG DNA varies with $\mathrm{CpG}$ content. Genome Res. 24 May 2010 (doi:10.1101/gr.103283.109)

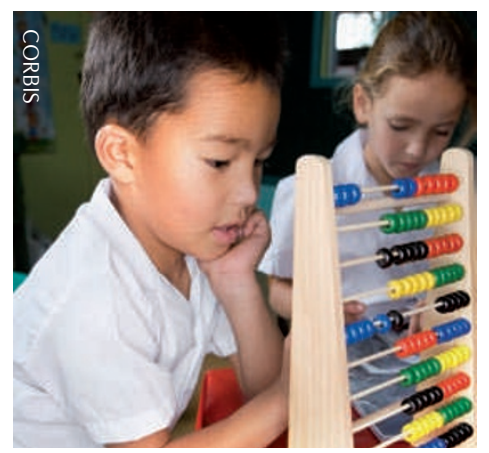

